\title{
Further Results on Multicarrier MFSK based Underwater Acoustic Communications
}

\author{
Xingyu Cai ${ }^{\mathrm{a}}$, Lei Wan ${ }^{\mathrm{b}}$, Yi Huang ${ }^{\mathrm{c}}$, Shengli Zhou ${ }^{\mathrm{c}, *}$, Zhijie Shi $^{\mathrm{a}}$ \\ ${ }^{a}$ Dept. of Computer Science and Engineering, University of Connecticut, Storrs, CT 06269, USA \\ ${ }^{b}$ Aquatic Sensor Network Technology (AquaSeNT), Storrs, CT 06268, USA \\ ${ }^{c}$ Dept. of Electrical and Computer Engineering, University of Connecticut, Storrs, CT 06269, USA
}

\begin{abstract}
Multicarrier $M$-ary frequency shift keying (MFSK), a parallel transmission of multiple MFSK data streams, is one basic reference scheme for underwater acoustic communications due to lowcomplexity incoherent processing at the receiver and ease of implementation. In this paper, we provide some further results for multicarrier MFSK based on the recent development of coherent orthogonal frequency division multiplexing (OFDM) schemes. Specifically, we adopt an OFDM based representation, develop a residual Doppler shift compensation approach at the receiver, and present different ways of computing the soft likelihood information for multicarrier MFSK transmissions in combination with nonbinary channel coding. As compared with coherent OFDM, simulation and semi-experimental results show that multicarrier MFSK has consistent performance in channels with different numbers of paths and in environments with different types of external noise.

Keywords: Underwater acoustic communications, OFDM, MFSK, impulsive noise, narrowband interference
\end{abstract}

\section{Introduction}

${ }^{*}$ Corresponding Author: Shengli Zhou, Department of Electrical and Computer Engineering, 371 Fairfield Way Unit-4157, University of Connecticut, Storrs, CT 06269-4157, USA; Email: shengli@engr.uconn.edu; Phone: 1-860-486-4593 
The underwater acoustic channel is commonly regarded as one of the most challenging channels for communication [1]. Lacking a widely adopted channel model, acoustic communication algorithms are often developed based on experimental data validation, and performance results from different experiments are often not directly comparable because the system performance depends on the site geometry and environmental conditions $[1,2]$. On the signal processing level, the major challenges are due to the large delay spread and fast time variation of the propagation paths $[1,2]$. Based on different principles, a variety of digital modulation schemes have been studied and tested for underwater acoustic communications over the past three decades $[1,3]$. Broadly speaking, they fall into two main categories, as illustrated in Fig. 1.

- Single carrier approaches. Direct sequence spread spectrum (DSSS) (e.g., $[4,5,6])$ as well as sweep-and-spread carrier (S2C) schemes [7] transmit information sequence in a signal band that is larger than the information rate. Lowering the spectrum efficiency much less than 1 bit per second per $\mathrm{Hz}$ (bit/s/Hz), robust performance could be achieved in low signal to noise ratio (SNR) applications or acoustic channels with a large number of propagation paths. Without spread spectrum operations, phase-coherent transmissions often operate at spectrum efficiency around one bits/s/Hz (e.g., $0.5 \sim 2$ bit/s/Hz). An effective equalizer is needed at the receiver side, and there are a plethora of receivers developed (e.g., $[8,9,10,11])$. Recently, multi-input multi-output (MIMO) techniques have been adopted to increase the spectrum efficiency to several bits/s/Hz (e.g., [12, 13, 14, 15]).

- Multiple carrier approaches. There are multiple frequency points, or subcarriers, available in the signal band. Frequency hopped frequency-shift-keying (FSK) hops a FSKmodulated symbol to a different frequency point at each symbol interval [16]. As a spread spectrum scheme, it has an efficiency much lower than 1 bit/s/Hz. Multiple FSK data streams could be transmitted in parallel to increase the data rate, where the center frequency of each data stream could be fixed or could hop within the signal band $[17,18,19]$. 


$\begin{array}{llll}\text { Single Carrier (SC): } & \text { DSSS, S2C } & \text { PSK/QAM } & \text { MIMO } \\ \text { Multicarrier (MC): } & \begin{array}{l}\text { FH-FSK } \\ \text { Multiple FSK }\end{array} & \begin{array}{c}\text { Noncoherent, Differential } \\ \text { or Coherent OFDM }\end{array} & \text { MIMO-OFDM } \\ { } &{\text { spectrum efficiency }}\end{array}$

Figure 1: The overview of underwater acoustic communication methods

Orthogonal-frequency division multiplexing (OFDM) uses closely-packed orthogonal subcarriers, where the superposition of parallel subcarriers is implemented based on the discrete Fourier transform. On top of OFDM, noncoherent modulations such as on-off keying (OOK) [20] and coherent modulations such as phase-shift-keying and quadratureamplitude-modulation (e.g., [21, 22, 23, 24, 25]) on each subcarrier, or differential modulation can be adopted across OFDM subcarriers [26, 27]. The spectrum efficiencies are typically around one or two bits/s/Hz. MIMO-OFDM further increases the spectrum efficiency to several bits/s/Hz through parallel transmissions in the spatial domain (e.g., $[28,29])$.

Note that different schemes have different characteristics on data rate, operational SNR ranges, and receiver complexities. Hence a particular application will have its own favorite among the available choices.

In this paper, we focus on the multicarrier MFSK scheme. Intuitively, the transmission consists of multiple $M$-ary FSK data streams in parallel, with each data stream located at a different center frequency $[17,18,19]$. Consistent with this intuitive idea, we choose to adopt the OFDM based implementation, where the subcarriers are overlapping but orthogonal to each other; hence another suitable name for this particular implementation is OFDM-MFSK. The key motivation for this paper is to leverage the insights learned from the progresses made on 
coherent OFDM (see e.g., [25] and references therein) to the transceiver design of the noncoherent multicarrier MFSK scheme. The specific contributions of this paper are as follows.

- We present a method for residual Doppler shift compensation at the receiver to reduce the intercarrier interference (ICI). Different from existing approaches, the proposed method does not require the existence of null subcarriers [22] or pilot tones [19].

- We evaluate the performance of the OFDM-MFSK scheme with nonbinary channel coding with two different ways to compute the log-likelihood ratio (LLR). Matching the size of the $M$-ary modulation with the size of Galois field avoids the bit to symbol mapping and vice versa. It also avoids the need to estimate the channel amplitude and the noise variance in one presented method of the LLR computation.

- We compare the performance of OFDM-MFSK with that of coherent OFDM in the presence of different types of external noises, including Gaussian noise, impulsive noise, and partial-band partial-block-duration noise. Performance results show that the OFDMMFSK scheme exhibited its consistency over OFDM-BPSK in two aspects: consistency against different numbers of paths in a multipath channel, and consistency over different types of external noise.

To our knowledge, these issues have not been explicitly addressed in the literature for the multicarrier MFSK scheme. The study in this paper hence contributes to further understanding of this legacy scheme.

The rest of the paper is organized as follows. Section 2 presents the transmitter design and the PAPR reduction method. Section 3 presents the receiver design, with the Doppler shift compensation and the coupling with nonbinary channel coding. Sections 4 and 5 contain simulation and experimental results, respectively. Conclusions are drawn in Section 6. 


\section{Transmitter Design}

In this paper, we adopt an implementation of multicarrier MFSK where overlapping OFDM subcarriers are used to carry MFSK symbols. The presentation here will follow the zero-padded (ZP) OFDM structure in [22]. Consider one OFDM block with symbol duration $T$, hence the subcarrier spacing is $1 / T$. Assume that there are $K$ subcarriers in total over a signal band $B=K / T$. The $k$ th subcarrier is located at the frequency

$$
f_{k}=f_{\mathrm{c}}+\frac{k}{T}, \quad k=-\frac{K}{2}, \ldots, \frac{K}{2}-1,
$$

where $f_{\mathrm{c}}$ is the center frequency. Let $T_{\mathrm{g}}$ denote the zero-padding interval and $T_{\mathrm{bl}}=T+T_{\mathrm{g}}$ the total block duration corresponding to one ZP-OFDM block. With $d[k]$ as the information symbol on the $k$ th subcarrier and $\phi[k]$ a phase rotation term to be specified later, the transmitted signal is:

$$
\tilde{x}(t)=\sum_{k=-K / 2}^{K / 2-1} e^{j \phi[k]} d[k] e^{j 2 \pi f_{k} t} g(t)
$$

where $g(t)$ is the rectangular pulse shaping filter: $g(t)=1 / T$ for $t \in[0, T]$ and $g(t)=0$ elsewhere.

Now, we specify how the information bits are mapped to the data symbols $\{d[k]\}$. Suppose that there are $N_{\mathrm{g}}$ parallel MFSK transmissions in one OFDM block and a channel code of rate $r_{\mathrm{c}}$ is used. If a binary code is used, $r_{\mathrm{c}} N_{\mathrm{g}} \log _{2} M$ information bits will be coded into $N_{\mathrm{g}} \log _{2} M$ bits, which will be divided into $N_{\mathrm{g}}$ groups. For the $i$ th group, let $\left\{l_{0}, \ldots, l_{M-1}\right\}$ denote the subcarrier indexes used by MFSK. Based on the value of $\log _{2} M$ coded bits, one of $d\left[l_{0}\right], \ldots, d\left[l_{M-1}\right]$ will be one and the rest will be zeros. In this paper, we adopt the nonbinary channel codes from [30]. Operating over Galois field $\mathrm{GF}(M), r_{\mathrm{c}} N_{\mathrm{g}}$ information symbols are mapped to $N_{\mathrm{g}}$ coded symbols, and each coded symbol is used to choose one frequency out of $M$ choices for transmission. Matching the size of the MFSK modulation with the size of Galois field avoids the bit to symbol mapping and vice versa. 
One practical issue is that OFDM-MFSK has large peak to average power ratio (PAPR) due to parallel transmissions, similar to coherent OFDM. The PAPR for each block is defined as:

$$
\operatorname{PAPR}=\frac{\max |\tilde{x}(t)|^{2}}{\frac{1}{T} \int_{0}^{T}|\tilde{x}(t)|^{2} d t}
$$

Since phase rotation at the transmitter side does not affect noncoherent processing at the receiver, the phases $\{\phi[k]\}$ can be adjusted to reduce the PAPR, i.e.,

$$
\{\hat{\phi}[k]\}_{\forall k}=\arg \min _{\{\phi[k]\}} \frac{\max \left|\sum_{-K / 2}^{K / 2-1} e^{j \phi[k]} d[k] e^{j 2 \pi f_{k} t}\right|^{2}}{\frac{1}{T} \int_{0}^{T}\left|\sum_{-K / 2}^{K / 2-1} e^{j \phi[k]} d[k] e^{j 2 \pi f_{k} t}\right|^{2} d t} .
$$

Optimizing the phase values for each OFDM block might be time consuming. Ref. [31] have compared different methods, including the selective mapping (SLM) method based on random search $[32,33,34]$, the time-frequency swapping method $[35,36]$, and the sequential algorithm developed in [31]. In this paper, we adopt the random search method based on the selected mapping principle due to its straightforward implementation. The transmitter randomly generates $\{\phi[k]\}_{\forall k}$ multiple times (say, $N$ times) and chooses the one with the smallest PAPR to transmit. Note that there is no side information that needs to be communicated to the receiver, hence there is no overhead.

With the OFDM-MFSK parameters to be specified in the simulation section, Fig. 2 shows the complementary cumulative density function $\operatorname{Pr}(\mathrm{PAPR}>x)$ with the random selection method on the passband data sampled at the sampling rate $f_{\mathrm{s}}=48 \mathrm{kHz}$. Here, the phase value on each subcarrier $\phi[k]$ is randomly selected from $[0,2 \pi]$. Repeat this procedure $N$ times and pick the one with smallest PAPR. Apparently, the PAPR reduction is very effective with a small number of searches; $N=8$ is chosen for the data sets used in the experiments.

Note that the PAPR control is applied on each block. If the transmitter is not operating in the full power mode, typically the average power of the OFDM blocks within a data burst can be 


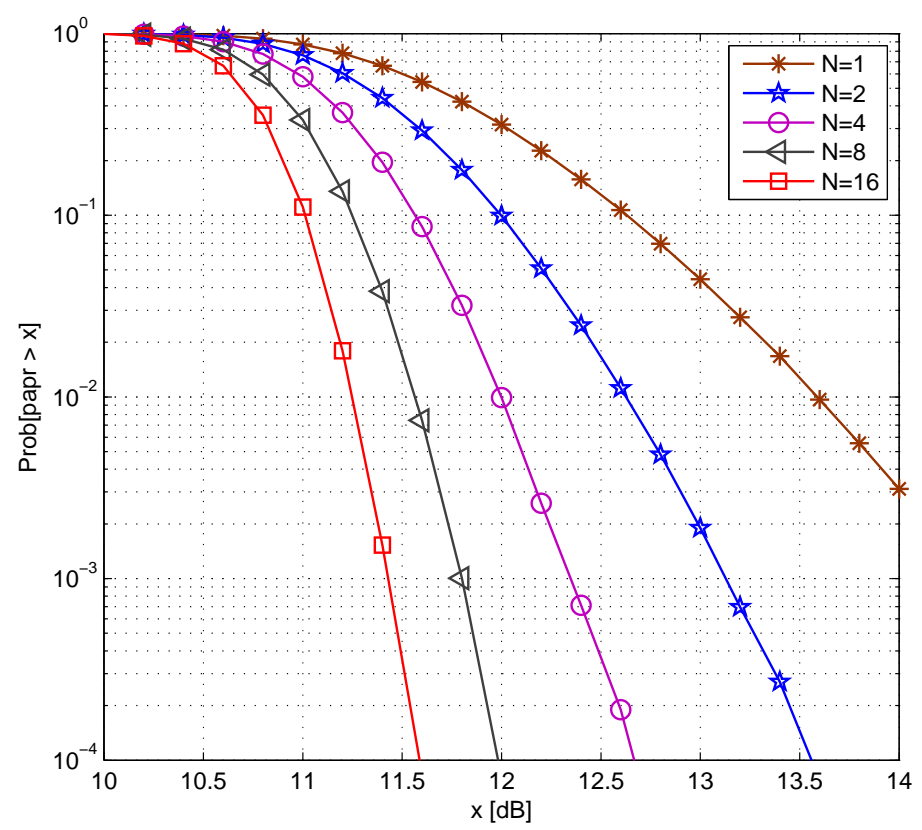

Figure 2: PAPR reduction for OFDM-FSK with the selected mapping algorithm

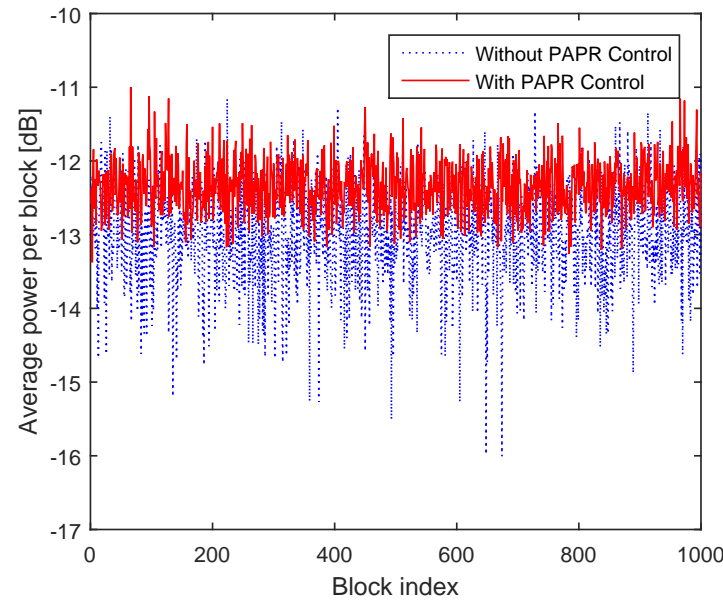

(a) Average power

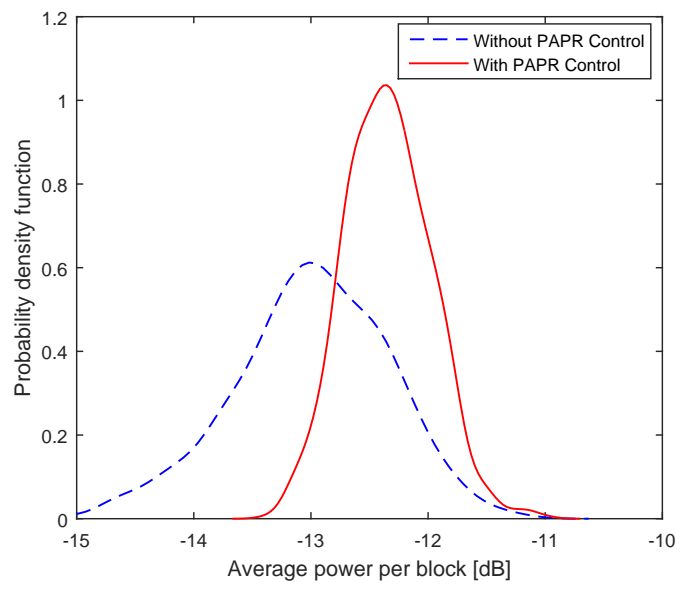

(b) Empirical pdf

Figure 3: The average power with a fixed peak power at 0dB for each block; 1000 blocks are shown. 
kept constant, and the peak power has slight variations from block to block. If the transmitter is operating in the full power mode, the peak power is often required to be strictly limited for hardware protection. In this case, the peak power of each OFDM block within a data bust is kept constant, and the average power has slight variations from block to block. Now, let us generate 1000 blocks, with the peak power fixed at $0 \mathrm{~dB}$ for each block, and show the average power variations in Fig. 3(a) and the empirical probability-density-function (pdf) in Fig. 3(b). It can be found with the per-block peak power fixed, the average power is varying within a 2 $\mathrm{dB}$ range when PAPR control is used, and the variation is much bigger if PAPR control is not applied. In this paper, we choose the approach to fix the peak power of all the blocks when preparing the data sets for experiments, as those data will be delivered to the field engineers with a possible full power transmission ${ }^{1}$.

\section{Receiver Design}

A generic time-varying multipath channel for underwater acoustic communications is expressed as

$$
h(t, \tau)=\sum_{p=1}^{N_{\mathrm{pa}}} A_{p} \delta\left(t-\left[\tau_{p}-a_{p} t\right]\right),
$$

where $N_{\text {pa }}$ is the number of paths, $A_{p}, \tau_{p}$, and $a_{p}$ are the amplitude, delay, and Doppler scale for the $p$ th path, respectively [37]. The received passband signal is

$$
\begin{aligned}
\tilde{y}(t) & =h(t, \tau) \star \tilde{x}(t)+\tilde{w}(t) \\
& =\sum_{p=1}^{N_{\mathrm{pa}}} A_{p} \tilde{x}\left(\left(1+a_{p}\right) t-\tau_{p}\right)+\tilde{w}(t),
\end{aligned}
$$

\footnotetext{
${ }^{1}$ Note that the average signal-to-noise ratios for all the OFDM blocks can be adjusted to the same level in offline data processing by scaling the added noise on each block properly. This is done in Sections 5.1 and 5.2.
} 
where $\tilde{w}(t)$ is the additive noise.

Often the Doppler scales $\left\{a_{p}\right\}$ are assumed to be concentrated around a mean, which can be estimated e.g., through a preamble preceding the data blocks [38] or the joint use of a preamble and a postamble [22]. Denote the estimated Doppler scale as $\hat{a}$. The main Doppler effect is removed through a resampling step, leading to the resampled signal

$$
\tilde{z}(t)=\tilde{y}\left(\frac{t}{1+\hat{a}}\right)
$$

If the resampling step is not used, then $\tilde{z}(t)=\tilde{y}(t)$. The passband signal is shift to baseband

as $z(t)=\operatorname{LPF}\left(\tilde{z}(t) e^{-j 2 \pi f_{\mathrm{c}} t}\right)$, where LPF stands for low pass filtering. The baseband signal is sampled at the baseband rate to obtain:

$$
z[n]=\left.z(t)\right|_{t=\frac{n T}{K}}, \quad n=0,1, \ldots, K+L-1
$$

where $L=\left\lfloor\frac{K T_{\mathrm{g}}}{T}\right\rfloor$.

\subsection{Residual Doppler Shift Compensation}

The residual Doppler shift after the resampling step can still be a major factor to limit the system performance. In [22], null subcarriers have been inserted in each OFDM block to facilitate the residual Doppler shift estimation. For OFDM-MFSK, residual Doppler shift estimation can be carried out without the use of null subcarriers.

Assume that the residual Doppler shift is the same for all subcarriers, and is in the range of $\left[-\frac{\Delta f}{2}, \frac{\Delta f}{2}\right]$. Collect the time-domain samples into a vector $\mathbf{z}=[z[0], \ldots z[K+L-1]]^{T}$, and Doppler shift compensation is performed in the time domain before frequency-domain samples are obtained. For clarify of presentation, first define a diagonal matrix as

$$
\boldsymbol{\Lambda}(\epsilon):=\operatorname{diag}\left(1, e^{j 2 \pi \epsilon / B}, \cdots, e^{j 2 \pi \epsilon(K+L-1) / B}\right) .
$$


Then define a matrix $\mathbf{O}=\left[\begin{array}{ll}\mathbf{I}_{K} & \mathbf{I}_{L}\end{array}\right]$ that performs an overlap-add operation on a vector, where $\mathbf{I}_{L}$ is the first $L$ columns of $\mathbf{I}_{K}$. Finally define a $K \times K$ Fourier transform matrix $\mathbf{F}$ with the $(p+1, q+1)$ entry $e^{-j 2 \pi p q / K}$. A one-dimensional search can be adopted to estimate $\epsilon$ as:

$$
\hat{\epsilon}=\underset{\epsilon}{\arg \max }\left\{\sum_{l=0}^{N_{\mathrm{g}}-1} \max _{0 \leq i \leq M-1}[\mathbf{Z}(\epsilon)]_{l_{i}}\right\}
$$

where $l_{i}$ is the index corresponding to the $i$ th frequency of the $l$ th group and the frequencydomain vector is evaluated as

$$
\mathbf{Z}(\epsilon)=\mathbf{F O} \Lambda^{*}(\epsilon) \mathbf{z}
$$

Instead of minimizing the energy on the null subcarriers as in [22], here the maximum energy is sought on tentative MFSK frequencies to determine the residual Doppler shift. Also, unlike the traditional method as in [19], the proposed method does not require the insertion of pilot tones.

\subsection{Log-Likelihood Ratio Calculation}

After Doppler shift compensation, the equivalent channel input and output model for the $l$ th group is

$$
Z\left[l_{i}\right]=H\left[l_{i}\right] e^{j \phi\left[l_{i}\right]} d\left[l_{i}\right]+n\left[l_{i}\right], \quad i=0, \ldots, M-1
$$

The receiver needs to generate soft information for the nonbinary channel decoder. Specifically, a vector

$$
\mathbf{L}[l]=\left[\operatorname{LLR}_{0}[l], \ldots, \operatorname{LLR}_{M-1}[l]\right]^{T}
$$

of the log-likelihood ratios (LLRs) is needed, where the LLR is defined as

$$
\operatorname{LLR}_{i}[l]=\ln \frac{\operatorname{Pr}\left(\left\{Z\left[l_{i}\right]\right\}_{i=0}^{M-1} \mid d\left[l_{i}\right]=1\right)}{\left.\operatorname{Pr}\left(\left\{Z\left[l_{i}\right]\right\}_{i=0}^{M-1} \mid d\left[l_{0}\right]=1\right\}\right)}
$$


First, we consider the case where the receiver does not attempt to acquire the channel amplitude information. Instead, the receiver views $\left|Z\left[l_{i}\right]\right|$ as Rayleigh distributed with variance $\sigma_{\mathrm{H}}^{2}[l]+\sigma_{\mathrm{n}}^{2}[l]$ if $d\left[l_{i}\right]=1$, and Rayleigh distributed with variance $\sigma_{\mathrm{n}}^{2}[l]$ if $d\left[l_{i}\right]=0$. The LLR can be calculated as

$$
\operatorname{LLR}_{i}[l]=\frac{\sigma_{\mathrm{H}}^{2}[l]}{\sigma_{\mathrm{H}}^{2}[l]+\sigma_{\mathrm{n}}^{2}[l]} \frac{\left|Z\left[l_{i}\right]\right|^{2}-\left|Z\left[l_{0}\right]\right|^{2}}{\sigma_{\mathrm{n}}^{2}[l]} .
$$

Assume that the channel and noise variances are similar across all groups, one can adopt an approximate version as (after scaling by a constant)

$$
\operatorname{LLR}_{i}[l] \propto\left(\left|Z\left[l_{i}\right]\right|^{2}-\left|Z\left[l_{0}\right]\right|^{2}\right)
$$

This computation solely depends on the measurements on the subcarriers, and hence it could be a robust statistics under various conditions.

Further, we consider the possibility that estimation of the channel amplitude and the noise variance might help to improve the receiver performance. Among $M$ subcarriers in each group, only one subcarrier is used and there are noises on the rest $M-1$ subcarriers. The presence of a signal component most likely increases the energy on a subcarrier, and hence an estimate of the noise variance can be formed by:

$$
\hat{\sigma}_{\mathrm{n}}^{2}[l]=\alpha \min _{0 \leq i \leq M-1}\left|Z\left[l_{i}\right]\right|^{2}
$$

where based on the order statistics, $\alpha$ is chosen to be $M-1$ according to [39, eq. (5)]. A smoothing operation, or, local averaging, across multiple neighboring groups will be applied to obtain better estimates of the noise variance. In this paper, we assume that the noise is white, and thus the average of $\hat{\sigma}_{\mathrm{n}}^{2}[l]$ over all groups is taken as a refined noise variance estimate. The 
square of the channel amplitude on the $l$ th group $|\hat{H}[l]|^{2}$ is obtained as follows:

$$
|\hat{H}[l]|^{2}=\left(\max _{0 \leq i \leq M-1}\left|Z\left[l_{i}\right]\right|^{2}\right)-\hat{\sigma}_{\mathrm{n}}^{2}[l]
$$

A moving averaging operation can be applied on $|\hat{H}[l]|^{2}$ to smooth the noisy estimates.

With the channel amplitude $|\hat{H}[l]|$ and the noise variance $\hat{\sigma}_{\mathrm{n}}^{2}[l]$ available, $\left|Z\left[l_{i}\right]\right|$ is viewed as Rician distributed if $d\left[l_{i}\right]=1$, and Rayleigh distributed if $d\left[l_{i}\right]=0$. The LLR for the $l$ th MFSK group is computed as [40]:

$$
\operatorname{LLR}_{i}[l]=\ln \frac{I_{0}\left(2|\hat{H}[l]|\left|Z\left[l_{i}\right]\right| / \hat{\sigma}_{\mathrm{n}}^{2}[l]\right)}{I_{0}\left(2|\hat{H}[l]|\left|Z\left[l_{0}\right]\right| / \hat{\sigma}_{\mathrm{n}}^{2}[l]\right)}
$$

where $I_{0}(\cdot)$ is the 0th-order modified Bessel function of the first kind.

\section{Simulation Results}

In this section, we investigate the performance of the OFDM-MFSK scheme under different settings, and compare it with a coherent OFDM with BPSK modulation, where the total transmission power and the data rate are set to be the same.

Assume that there are a total of $K=1024$ OFDM subcarriers within a total bandwidth of $B=6 \mathrm{kHz}$, hence, the subcarrier spacing is $B / K=5.86 \mathrm{~Hz}$ and the OFDM symbol duration is $T=K / B=170.7 \mathrm{~ms}$. The carrier frequency $f_{\mathrm{c}}$ can be shifted to match with the transducer used in a practical system. It is selected to be $f_{\mathrm{c}}=17 \mathrm{kHz}$ in our simulation and experimental results. For the OFDM-MFSK scheme, only the middle $K_{\mathrm{d}}=672$ subcarriers are used, and are divided into 168 groups with four subcarriers in each group to accommodate a 4-FSK modulation. Nonbinary rate-1/2 LDPC coding over GF(4) is used. Two settings are considered. In the first setting, $84 \mathrm{GF}(4)$ source symbols are coded into $168 \mathrm{GF}(4)$ symbols. In the second case, 42 GF(4) source symbols are coded into $84 \mathrm{GF}(4)$ symbols, which are then repeated to form 168 
symbols (a repetition redundancy with a factor of 2). In both cases, there are $168 \mathrm{GF}(4)$ coded symbols that are modulated on the 168 subcarrier groups with 4-FSK modulation. Consider a guard interval $T_{\mathrm{g}}=79.3 \mathrm{~ms}$, the data rates for the two settings are

$$
\begin{aligned}
& R_{1}=\frac{84 \cdot \log _{2} 4}{T+T_{\mathrm{g}}}=672 \mathrm{bps}, \\
& R_{2}=\frac{42 \cdot \log _{2} 4}{T+T_{\mathrm{g}}}=336 \mathrm{bps} .
\end{aligned}
$$

For the OFDM-BPSK scheme, the number of data subcarriers is $K_{\mathrm{d}}=672$, the number of pilot subcarriers is $K_{\mathrm{p}}=256$, and the number of null subcarriers is $K_{\mathrm{n}}=96$ [22]. There are 24 null subcarriers on each edge of the frequency band, and 48 null subcarriers are mixed with data and pilot subcarriers. Similarly, two cases are considered. In the first case, 84 GF(4) source symbols are coded into $168 \mathrm{GF}(4)$ symbols, followed by a repetition with a factor 2 to generate $336 \mathrm{GF}(4)$ symbols. In the second case, $42 \mathrm{GF}(4)$ source symbols are coded into $84 \mathrm{GF}(4)$ symbols, which are then repeated four times to generate $336 \mathrm{GF}(4)$ symbols. In both cases, there are $336 \mathrm{GF}(4)$ symbols that can be mapped to 672 BPSK symbols to be loaded to the 672 data subcarriers. Hence, the same data rates of $R_{1}$ and $R_{2}$ are achieved. For clarification, these parameters are summarized in Table 1.

Note that the effective bandwidth for the OFDM-MFSK scheme is only $4 \mathrm{kHz}$ as only the middle 672 subcarriers are used, while it is around $6 \mathrm{kHz}$ for the OFDM-BPSK scheme. To make a reasonable comparison, all the performance plots will be evaluated based on the input signal to noise ratio defined over the $6 \mathrm{kHz}$ bandwidth:

$$
\text { Input } \mathrm{SNR}=\frac{\text { Signal power within the } 6 \mathrm{kHz} \text { signal band }}{\text { Noise power within the } 6 \mathrm{kHz} \text { signal band }}
$$

That is, OFDM-MFSK and OFDM-BPSK are compared with the same transmission power and the noise level. Or, one can say that these two schemes are compared with the same energy 
Table 1: List of the system parameters for the OFDM-MFSK and OFDM-BPSK schemes

\begin{tabular}{|l|l|}
\hline Common & $\begin{array}{l}\text { 1024 OFDM subcarriers, total system bandwidth } 6 \mathrm{kHz} \\
\text { Subcarrier spacing: } 6000 / 1024=5.86 \mathrm{~Hz}\end{array}$ \\
& OFDM symbol duration $170.7 \mathrm{~ms}$, guard time $79.7 \mathrm{~ms}$ \\
\hline OFDM-MFSK & 672 data subcarriers in the middle of the band \\
& Effective bandwidth $3.94 \mathrm{kHz}$ \\
& Rate $R_{1}=672 \mathrm{bps:} 84 \times 168 \mathrm{LDPC}$ over $\mathrm{GF}(4)$, no repetition \\
& Rate $R_{2}=336$ bps: $42 \times 84 \mathrm{LDPC}$ over $\mathrm{GF}(4)$, repetition by two \\
\hline OFDM-BPSK & 672 data subcarriers, 256 pilot subcarriers, 96 null subcarriers \\
& Effective bandwidth $5.72 \mathrm{kHz}$. \\
& Rate $R_{1}=672 \mathrm{bps}: 84 \times 168 \mathrm{LDPC}$ over $\mathrm{GF}(4)$, repetition by two \\
& Rate $R_{2}=336 \mathrm{bps}: 42 \times 84 \mathrm{LDPC}$ over $\mathrm{GF}(4)$, repetition by four \\
\hline
\end{tabular}

per information bit used. The block error rate (BLER) after channel decoding is used as the performance metric.

The passband data sets are generated with a sampling rate $f_{\mathrm{s}}=48 \mathrm{kHz}$. The inter-arrivaltime of paths follows an exponential distribution with a mean of $1 \mathrm{~ms}$. The amplitudes of paths are Gaussian distributed with the average power decreasing exponentially with the delay, where the difference between the beginning and the end of the guard time is $20 \mathrm{~dB}$. For each path, the Doppler scale $a_{p}$ is generated from a Doppler speed $v_{p}$ (with unit $\mathrm{m} / \mathrm{s}$ )

$$
a_{p}=v_{p} / c
$$

where $c=1500 \mathrm{~m} / \mathrm{s}$ is the sound speed in water. The values of the Doppler speed will be specified in each test case individually. Note that a block fading assumption is used, where each realization of the multipath channel is applied on a short data burst of five OFDM symbols.

\subsection{Comparison between Two LLR Computation Methods}

The LLR computation in (16) (termed as method 1) does not require estimation of the channel amplitude while the computation in (19) (termed as method 2) requires estimation of the channel amplitude and noise variance. The channels are generated without Doppler, where 


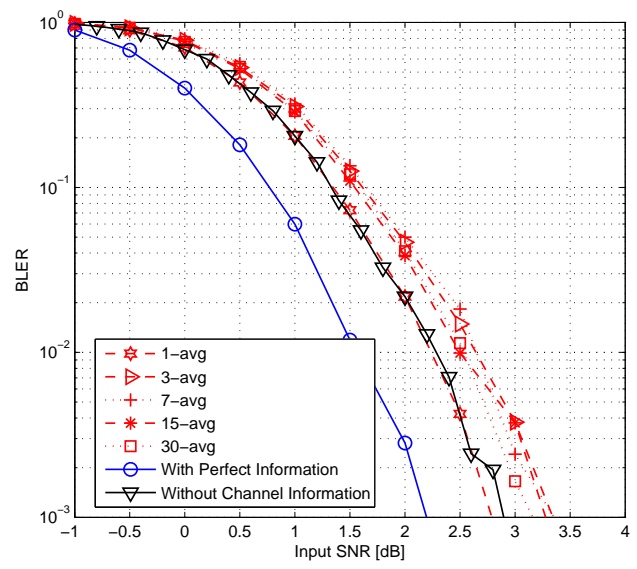

(a) Rate $R_{1}$

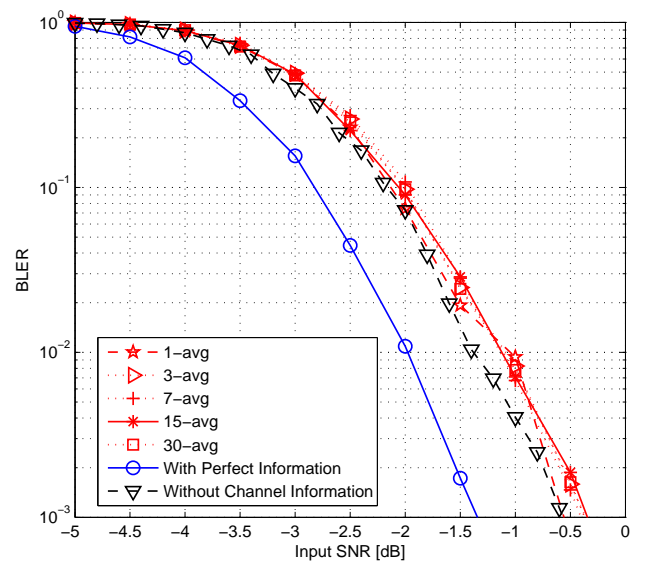

(b) Rate $R_{2}$

Figure 4: Comparison of the LLR computation methods for MFSK (30 paths, Gaussian noise).

$a_{p}=0, \forall p$. Fig. 4 compares the performance of both methods, where the channel amplitudes could be obtained through moving average with different window lengths. In the figure, " $n$-avg" means that the sliding average window has a size of $n$. Indeed, when the channel amplitude information is perfectly known, method 2 is better than method 1 . However, with estimated channel amplitudes, method 2 is not as good as method 1 while the latter is even much simpler. This is an encouraging observation that supports the adoption of method 1 in practical systems.

The following simulation and semi-experimental results are all performed using method 1 which does not need the knowledge of the channel amplitude and noise variance.

\subsection{Doppler Shift Compensation Effect on OFDM-MFSK}

We now enforce that all the channel paths have the same Doppler speed, $v_{p}=v, \forall p$, and compare the system performance with and without residual Doppler shift compensation. Fig. 5 shows the simulation result when $v=0.1,0.15,0.2 \mathrm{~m} / \mathrm{s}$ and non-Doppler case $(v=0)$ respectively, under the 10-path channel condition.

Note that the resampling step in (7) is intentionally switched off in this investigation; otherwise the common Doppler scale could be corrected by the resampling step. Clearly residual 


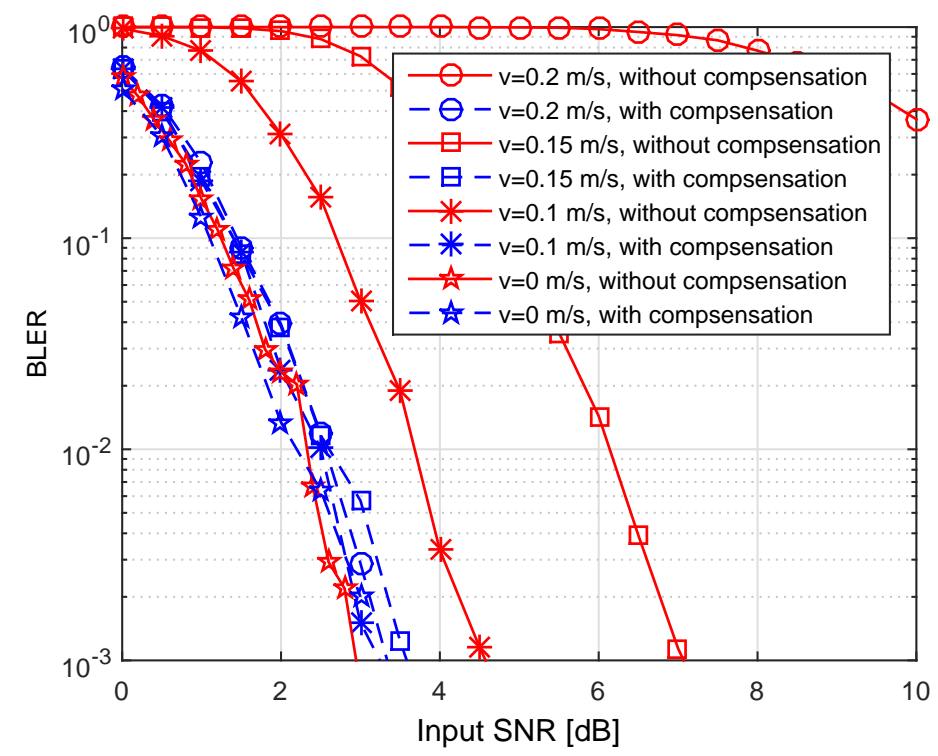

Figure 5: Performance comparison with and without Doppler shift compensation under a 10-path Channel

Doppler shift compensation plays an important role in delivering satisfactory performance. As Doppler speed increases, the receiver without Doppler compensation suffers significant drop of performance, while the one with Doppler compensation is nearly not affected.

\subsection{Performance Comparison for Multi-path Channel under Different Types of Noise}

Now we compare the performance of OFDM-MFSK and OFDM-BPSK in the presence of different types of noises, specifically, white Gaussian noise, impulsive noise and partial-band partial-block-duration interference. The simulation is performed using 10, 30 and 60-path conditions. As a reference, the performance with the AWGN channel is also included. Figs. 6 (a) and (b) show the performance comparison for both schemes with white Gaussian noise.

Due to human activities in close-to-harbor areas, impulsive noise exists widely and cannot be ignored as sometimes it even becomes the dominant interference in a sea environment. Impulsive noise behaves quite differently from Gaussian noise in terms of the large amplitude but very short duration. We are interested in how both schemes behave in environments with impulse noises. 
For each data block, we fix a number of 20 impulses with similar amplitude to be generated and placed randomly into the block. Also a background white Gaussian noise $20 \mathrm{~dB}$ lower than the signal is added to each data block. Figs. 6 (c) and (d) show the performance of the two schemes with impulsive noises.

We also investigate the performance under partial-band interference whose duration is less than the block duration $T$. In real scenarios, narrow band interference or jamming occurs frequently, e.g., due to concurrent sonar operations, or marine mammals. The work in [43] has explicitly dealt with the partial-band partial-block-duration interference in a coherent OFDM system. Here, small pieces of the LFM (Linear Frequency Modulation) signal are generated as the interference source. The LFM signal would spread $2 \mathrm{kHz}$ in the middle of the passband of $6 \mathrm{kHz}$. The time duration of the LFM signal lasts $40 \mathrm{~ms}$, which takes around $13 \%$ of total block duration. The interference is placed at a random position in the data block, along with a Gaussian background noise $20 \mathrm{~dB}$ below the useful signal. Figs. 6 (e) and (f) show the performance comparison of two schemes in presence of partial-band interference.

From Fig. 6, we have the following observations.

- As the number of paths increases, the performance of OFDM-MFSK improves while the performance of OFDM-BPSK deteriorates. Since channel estimation is not needed for OFDM-MFSK, the performance improvement could be attributed to the increased frequency diversity when the number of paths increases. The performance of OFDM-BPSK deteriorates due to the increased channel estimation error when the number of unknowns increases. 


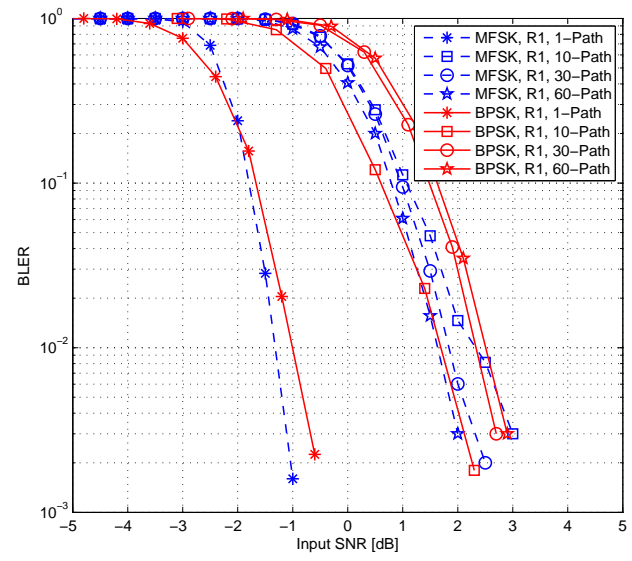

(a) MFSK R1 vs BPSK R1, Gaussian Noise

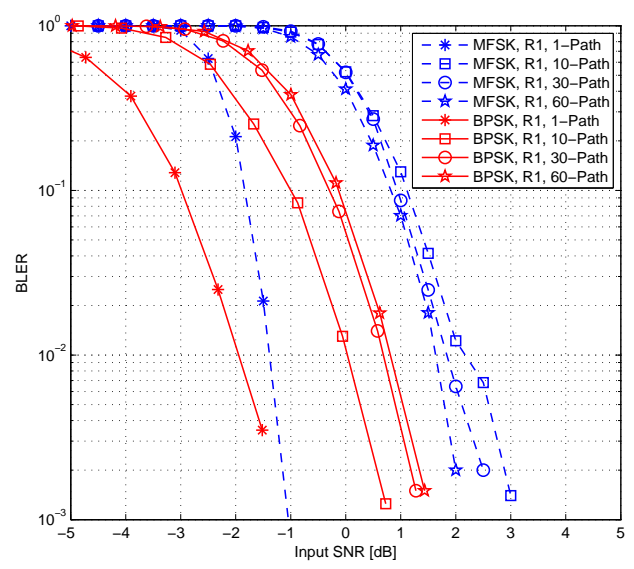

(c) MFSK R1 vs BPSK R1, Impulsive Noise

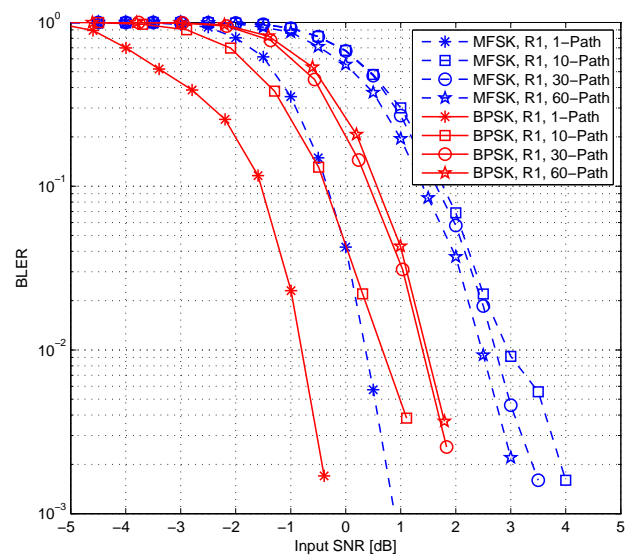

(e) MFSK R1 vs BPSK R1, Partial-band Noise

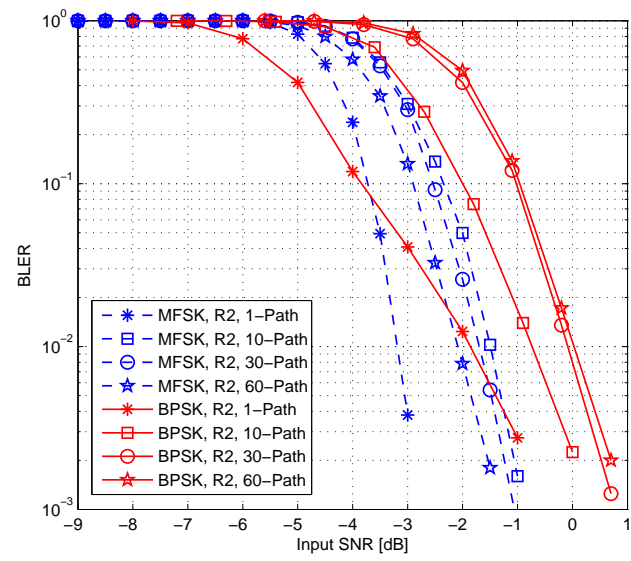

(b) MFSK R2 vs BPSK R2, Gaussian Noise

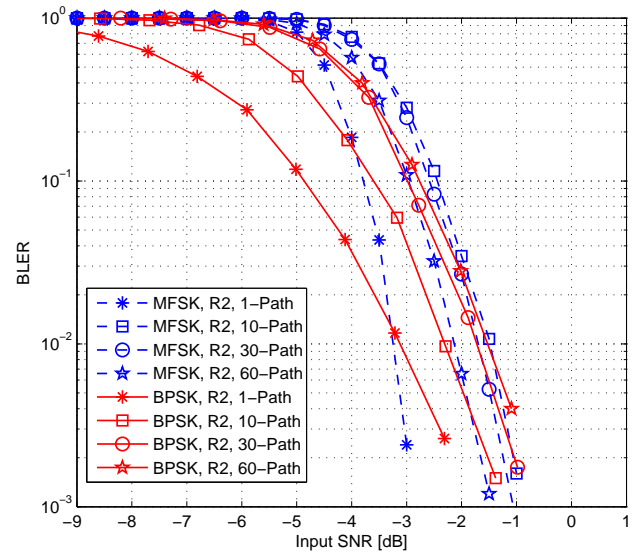

(d) MFSK R2 vs BPSK R2, Impulsive Noise

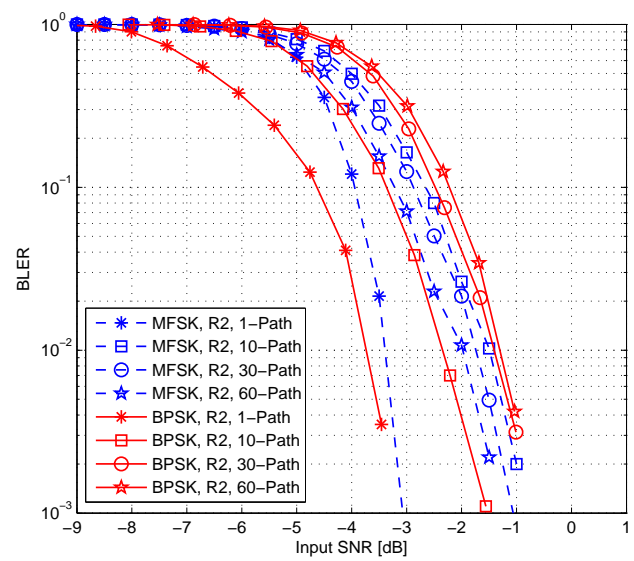

(f) MFSK R2 vs BPSK R2, Partial-band Noise 18

Figure 6: Performance comparison under multipath channels 
- No matter for the high data rate $\left(R_{1}\right)$ or the low data rate $\left(R_{2}\right)$, OFDM-MFSK shows more consistent performance across different numbers of paths. Specifically, the curves for OFDM-BPSK span a wider range for 10, 30 and 60 paths, where the curves for OFDMMFSK are closely spaced. This agrees with the intuition that OFDM-MFSK does not require channel estimation, and hence is less sensitive to the number of channel parameters to be estimated.

- For the high data rate case $\left(R_{1}\right)$, OFDM-BPSK outperforms OFDM-MFSK in either the impulsive-noise or partial-band-interference scenario, indicating that OFDM-BPSK could achieve better performance. A possible reason is that the OFDM-BPSK scheme fully uses all $6 \mathrm{kHz}$ bandwidth while the OFDM-MFSK scheme only uses $4 \mathrm{kHz}$, which brings an advantage in a high data-rate setting. But for the low data rate, OFDM-MFSK shows performance advantages, especially in the presence of the Gaussian noise.

- There is another aspect of robustness for OFDM-MFSK against different types of noises. Fig. 7 further illustrates this argument by comparing the performance with different types of noise in a 30-path channel. Clearly, the curves for OFDM-MFSK are spaced much closer to each other than those for OFDM-BPSK.

In short, simulation results show that OFDM-MFSK is a reliable scheme whose performance is not sensitive to the channel and noise characteristics.

\section{Performance Results with Experimental Data}

\subsection{Experiment Data from A Swimming Pool}

In this section, we present performance comparison of OFDM-MFSK and OFDM-BPSK schemes with experimental data sets collected from a swimming pool. Two AquaSeNT modems [41] have been used, one playing the pre-stored passband waveform files and the other recording 


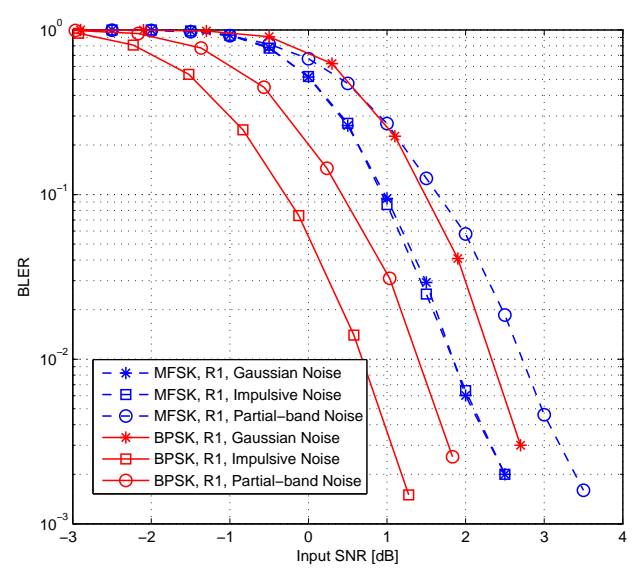

(a) MFSK $R_{1}$ vs BPSK $R_{1}$ in 30-path Channel

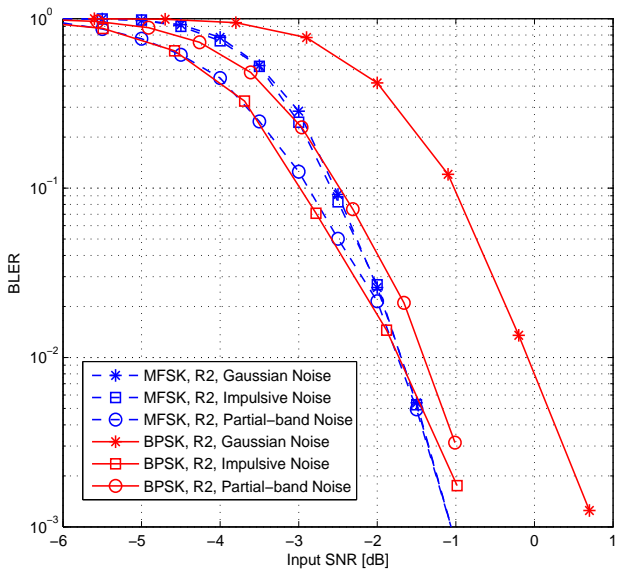

(b) MFSK $R_{2}$ vs BPSK $R_{2}$ in 30-path Channel

Figure 7: Performance comparison with different types of noise

the incoming data. The parameter settings are kept consistent with the simulation part, where the sampling rate is $f_{\mathrm{s}}=48 \mathrm{kHz}$, the center frequency is $f_{\mathrm{c}}=17 \mathrm{kHz}$, the data block duration is $T+T_{\mathrm{g}}=250 \mathrm{~ms}$, and two different rates $R_{1}=672 \mathrm{bps}$ and $R_{2}=336 \mathrm{bps}$ are considered. Each burst of transmission consists of 5 blocks of the MFSK $R_{1}$ data, followed by 5 blocks of the MFSK $R_{2}$ data, followed by 5 blocks of the BPSK $R_{1}$ data, and then 5 blocks of BPSK $R_{2}$. This way, each recorded data set contains different types of data blocks together, which shall experience the same or very similar channels. The transmitter and the receiver are 25 meters away, and hundreds of data sets were collected. An estimated channel response is shown in Fig. 8, where the delay spread is more than $30 \mathrm{~ms}$.

Now we add different types of recoded noises to the data sets. The Gaussian-type environmental noise was recorded in the swimming pool. The impulsive noise data sets were collected from the experiment conducted in May 2013, Kaohsiung, Taiwan, as used in [42], and the partialband Sonar interference data sets were recorded in the experiment carried out in March 2010, Atlantic Undersea Test and Evaluation Center (AUTEC), Andros Island near the Tongue of the Ocean, Bahamas, as used in [43]. Fig. 9 (a) shows an example of the impulse noise in a 


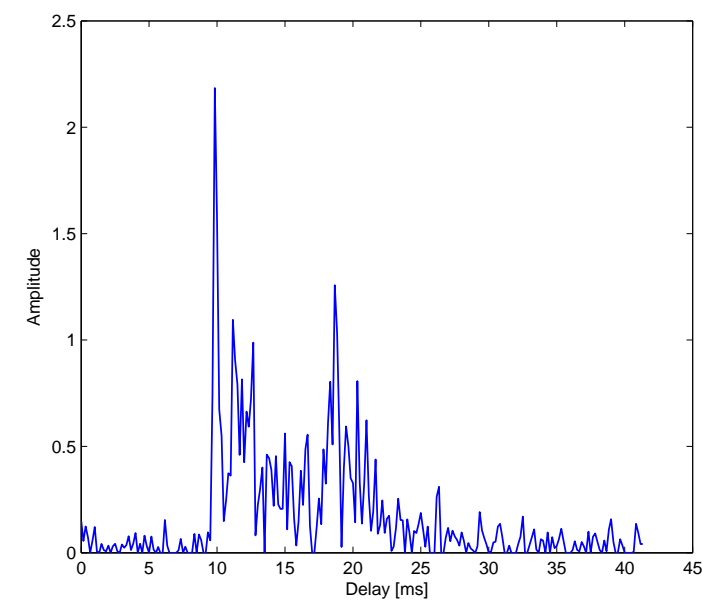

Figure 8: One estimated channel impulse response from the pool data

one-minute time duration, and Fig. 9 (b) shows the partial band Sonar interference, appearing about every other second. For both impulsive data and partial-band noise data, we pre-process them by cutting them into pieces of $100 \mathrm{~ms}$ per piece. When dealing with Gaussian noise, each OFDM block of duration $250 \mathrm{~ms}$ will be added to a noise block of $250 \mathrm{~ms}$. When dealing with the impulsive noise or the partial-band noise, the noise segments of $100 \mathrm{~ms}$ will be scaled to a different amplitude and added to the OFDM data blocks at random position within the block.

Fig. 10 shows the performance results as a function of the input SNR, in the presence of three different types of noises. The pool environment is stable, and the Doppler shift compensation has negligible effect on the performance. We clearly see that OFDM-MFSK exhibits more consistent performance in different types of noise. This observation agrees well with that from the simulation results.

\subsection{Experimental Data from Lake Superior}

An experiment was carried by a research group from the Michigan Technological University in the L'Anse Bay, Lake Superior, May 14, 2015. Our data sets as used in the swimming pool tests have been transmitted and recorded using AquaSeNT modems. We will use the data sets 


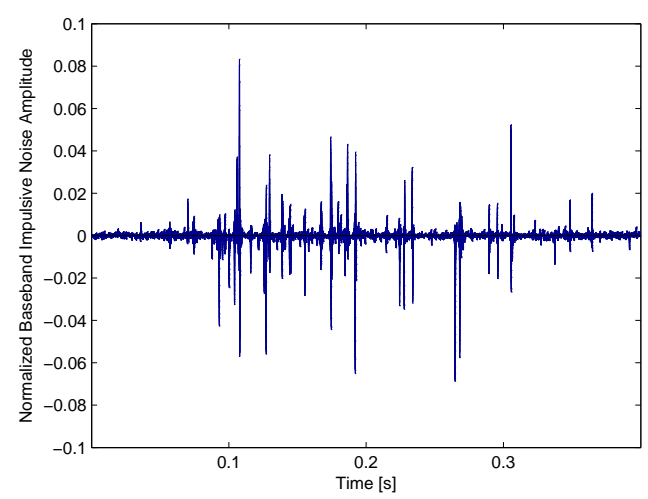

(a) Impulsive Noise Sample
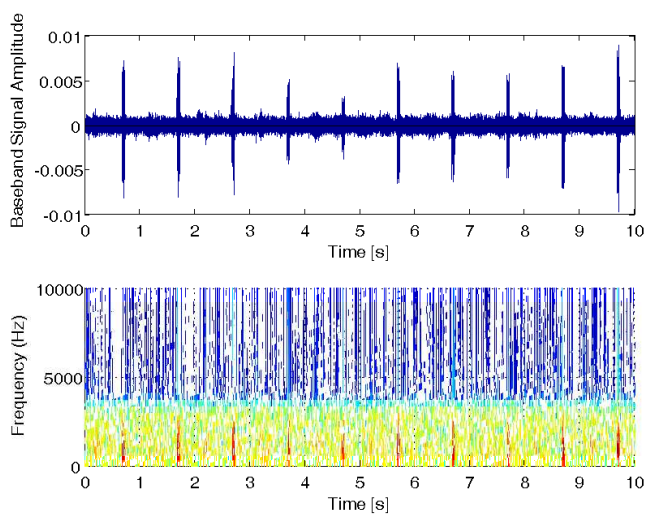

(b) Partial-band Noise Sample

Figure 9: Samples of the impulsive noise and the partial-band interference

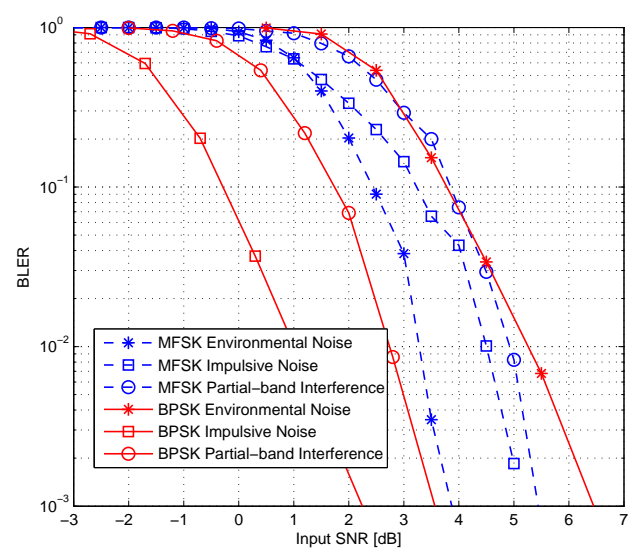

(a) $R_{1}$ Mode

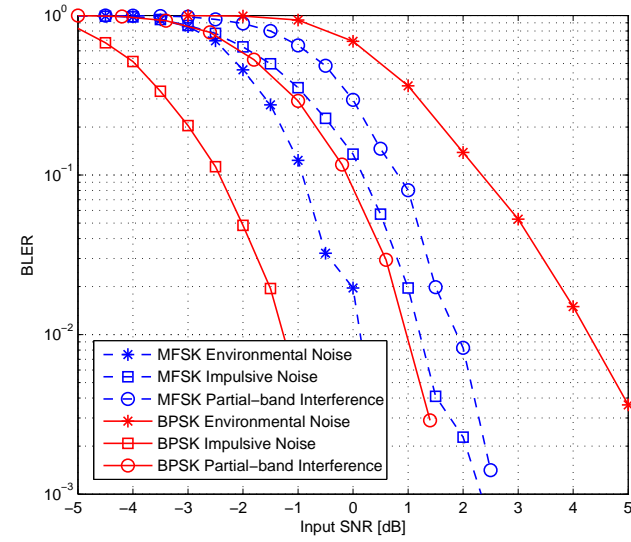

(b) $R_{2}$ Mode

Figure 10: The performance using the recorded data from a swimming pool mixed with different types of noise

collected at two distances, $460 \mathrm{~m}$ and $2000 \mathrm{~m}$, where the transmitter and the receiver were stationary in both cases. Table 2 shows the exact locations of the transmitter and the receiver, the water depths and the depths of the deployed modems. 


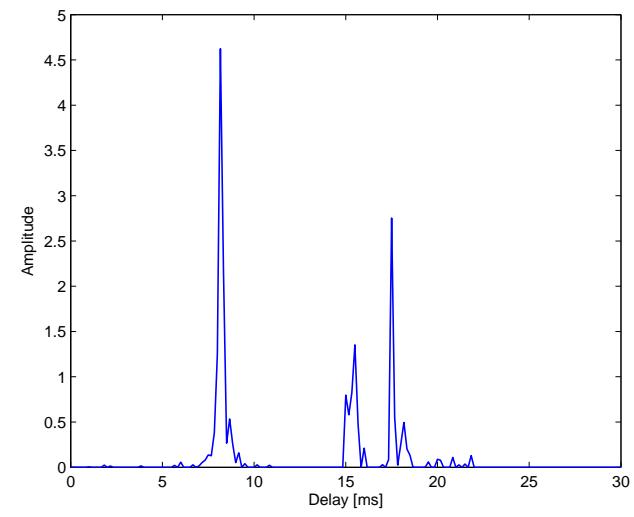

(a) $460 \mathrm{~m}$

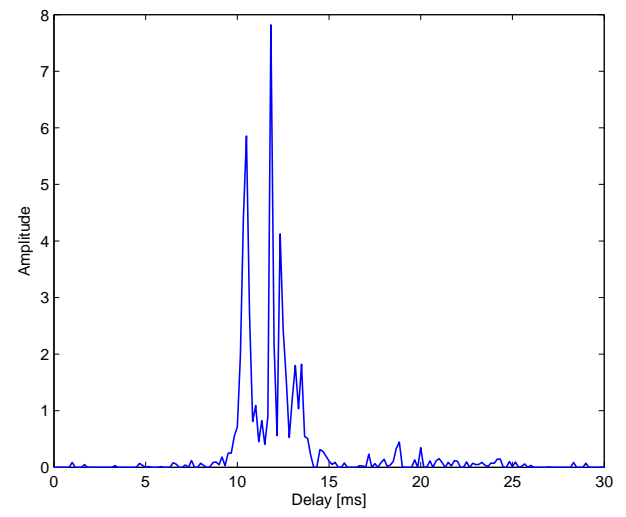

(b) $2000 \mathrm{~m}$

Figure 11: The example channel impulse responses from the lake data

Table 2: L'Anse Bay Experiment Settings

\begin{tabular}{|c|c|c|c|c|}
\hline Distance [m] & Latitude & Longitude & Water depth [m] & Modem depth [m] \\
\hline 0 (Transmitter) & $46^{\circ} 47^{\prime} 3.40^{\prime \prime}$ & $-88^{\circ} 27^{\prime} 17.65^{\prime \prime}$ & 54.98 & 8 \\
460 & $46^{\circ} 47^{\prime} 17.82^{\prime \prime}$ & $-88^{\circ} 27^{\prime} 11.80^{\prime \prime}$ & 54.14 & 8 \\
2000 & $46^{\circ} 48^{\prime} 6.39^{\prime \prime}$ & $-88^{\circ} 26^{\prime} 55.50^{\prime \prime}$ & 74.11 & 8 \\
\hline
\end{tabular}

Fig. 11 shows the example channel impulse responses at two distances, where the channel clusters (from e.g., direct paths, surface bounces and bottom bounces) of the channel at $460 \mathrm{~m}$ are more spread out than those at 2000 m, as expected based on the geometry. Even though the transmitter and the receiver were stationary, Fig. 12 shows that Doppler shift compensation can improve the system performance by 0.5 to $1 \mathrm{~dB}$ in terms of input SNRs, where the recorded environmental noise was added to the data sets.

Three types of noise (environmental, impulsive noise and partial-band interference) are added for performance comparison, where the impulsive noise and partial-band interference are the same as used in Section 5.1. Fig. 13 shows the consistency of OFDM-MFSK in the presence of different types of noises. Also, at the low rate setting, OFDM-MFSK outperforms OFDM-BPSK considerably in the presence of environmental noise. 


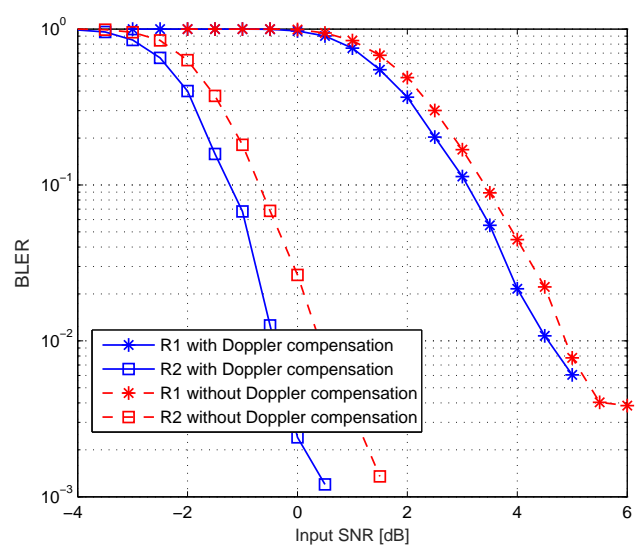

(a) $460 \mathrm{~m}$

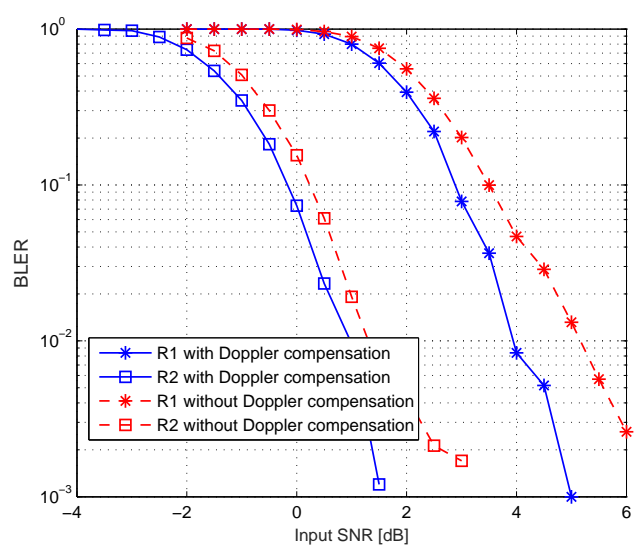

(b) $2000 \mathrm{~m}$

Figure 12: The effect of Doppler shift compensation, data collected from the L'Anse Bay

\section{Conclusions}

This paper presented several improvements for the OFDM-MFSK modulation (a.k.a. multicarrier MFSK), where multiple MFSK symbols are carried by the OFDM subcarriers. The selective mapping approach can effectively reduce the PAPR at the transmitter, and the proposed Doppler shift compensation estimation method can mitigate the Doppler effect at the receiver. Comparisons were carried out between two different likelihood-ratio computation methods, which showed that the low-complexity approach is in fact better without trying to estimate the channel amplitudes.

Using both simulation and experimental data sets, we carried out comprehensive comparisons between OFDM-MFSK and OFDM-BPSK subject to the same data rate and the same energy per bit, but with different bandwidths and receiver complexity. The OFDM-MFSK scheme exhibited its consistency over OFDM-BPSK in two aspects: consistency against different numbers of paths in a multipath channel, and consistency over different types of external noise. 


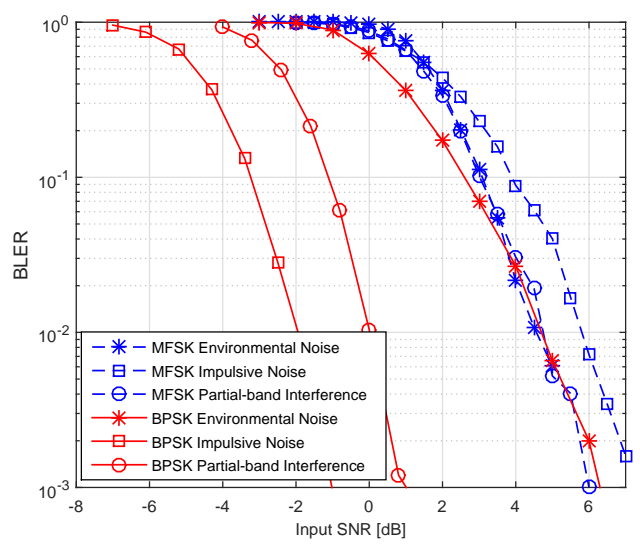

(a) Rate $R_{1}, 460 \mathrm{~m}$

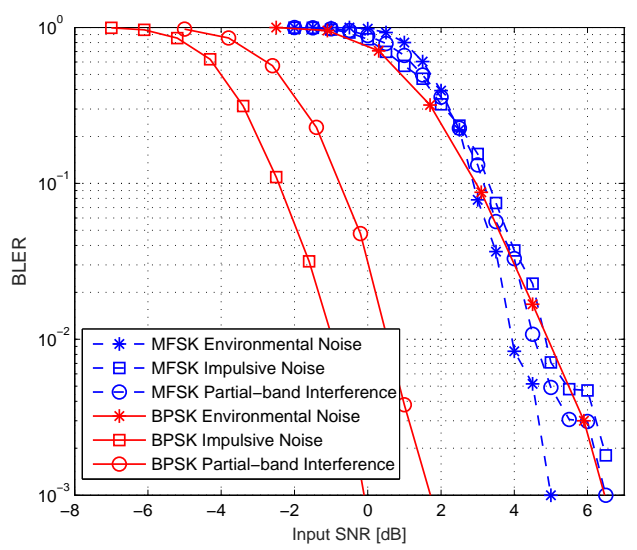

(c) Rate $R_{1}, 2000 \mathrm{~m}$

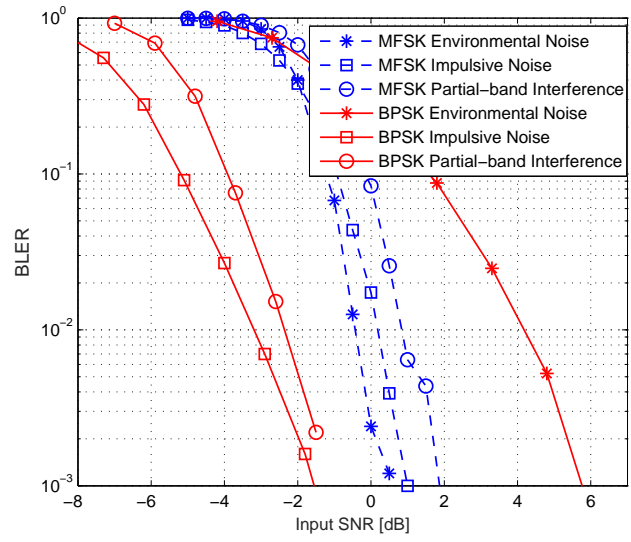

(b) Rate $R_{2}, 460 \mathrm{~m}$

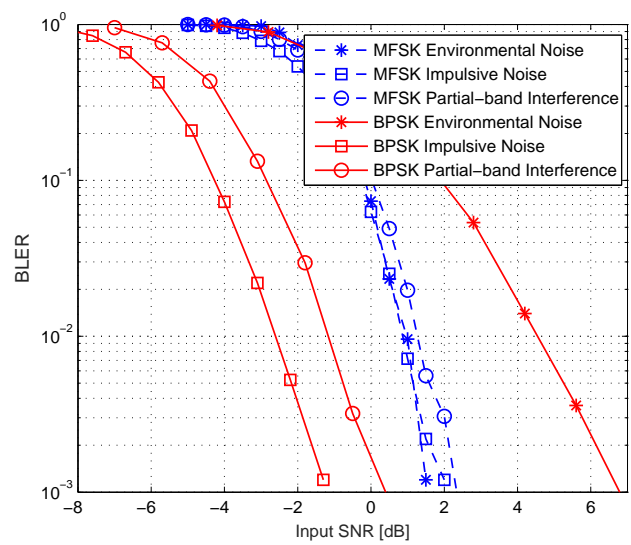

(d) Rate $R_{2}, 2000 \mathrm{~m}$

Figure 13: The performance using the recorded data from L'Anse Bay mixed with different types of noise

\section{Acknowledgement}

This work was supported by the NSF grants CNS-1228936, PLR-1344264, and ECCS1310406. The authors thank Dr. Zhaohui Wang, Dr. Guy Meadows and their teams from Michigan Technological University for collecting the data sets from the experiment in Lake Superior. 


\section{References}

[1] D. B. Kilfoyle and A. B. Baggeroer, "The state of the art in underwater acoustic telemetry," IEEE Journal of Oceanic Engineering, vol. 25, no. 1, pp. 4-27, Jan. 2000.

[2] M. Stojanovic and J. Preisig, "Underwater acoustic communication channels: Propagation models and statistical characterization," IEEE Communications Magazine, vol. 47, no. 1, pp. 84-89, Jan. 2009.

[3] A. B. Baggeroer, "An overview of acoustic communications from 2000-2012," in Proc. of the Workshop on Underwater Communications: Channel Modelling $\&$ Validation, Italy, Sep. 2012.

[4] L. Freitag, M. Stojanovic, S. Singh, and M. Johnson, "Analysis of channel effects on directsequence and frequency-hopped spread-spectrum acoustic communications," IEEE Journal of Oceanic Engineering, vol. 26, no. 4, pp. 586-593, Oct. 2001.

[5] T.-C. Yang and W.-B. Yang, "Performance analysis of direct-sequence spread-spectrum underwater acoustic communications with low signal-to-noise-ratio input signals," J. Acoust. Soc. Am., vol. 123, no. 2, pp. 842-855, Feb. 2008.

[6] Z. Liu, K. Yoo, T. C. Yang, S. E. Cho, H. C. Song, and D. E. Ensberg, "Long-range doubledifferentially coded spread-spectrum acoustic communications with a towed array," IEEE Journal of Oceanic Engineering, vol. 39, no. 3, pp. 482-490, Jul. 2014.

[7] K. Kebkal and R. Bannasch, "Sweep-spread carrier for underwater communication over acoustic channels with strong multipath propagation," J. Acoust. Soc. Am., vol. 112, no. 5, pp. 2043-2052, Nov. 2002.

[8] M. Stojanovic, J. A. Catipovic, and J. G. Proakis, "Phase-coherent digital communications for underwater acoustic channels," IEEE Journal of Oceanic Engineering, vol. 19, no. 1, 
pp. 100-111, Jan. 1994.

[9] H. C. Song, W. S. Hodgkiss, W. A. Kuperman, M. Stevenson, and T. Akal, "Improvement of time reversal communications using adaptive channel equalizers," IEEE J. Oceanic Eng., vol. 31, no. 2, pp. 487-496, Apr. 2006.

[10] A. Song, M. Badiey, A. E. Newhall, J. F. Lynch, H. A. DeFerrari, and B. G. Katsnelson, "Passive time reversal acoustic communications through shallow-water internal waves," IEEE J. Ocean. Eng., vol. 35, no. 4, pp. 756 -765, Oct. 2010.

[11] Y. R. Zheng, C. Xiao, T. C. Yang, and W. B. Yang, "Frequency-domain channel estimation and equalization for shallow-water acoustic communications," Elsevier Journal of Physical Communication, vol. 3, pp. 48-63, Mar. 2010.

[12] H. C. Song, P. Roux, W. S. Hodgkiss, W. A. Kuperman, T. Akal, and M. Stevenson, "Multiple-input/multiple-output coherent time reversal communications in a shallow water acoustic channel," IEEE Journal of Oceanic Engineering, vol. 31, no. 1, pp. 170-178, Jan. 2006 .

[13] S. Roy, T. M. Duman, V. McDonald, and J. G. Proakis, "High rate communication for underwater acoustic channels using multiple transmitters and space-time coding: Receiver structures and experimental results," IEEE Journal of Oceanic Engineering, vol. 32, no. 3, pp. 663-688, Jul. 2007.

[14] J. Tao, Y. R. Zheng, C. Xiao, and T. C. Yang, "Robust MIMO underwater acoustic communications using turbo block decision-feedback equalization," IEEE Journal of Oceanic Engineering, vol. 35, no. 4, pp. 948-960, Oct. 2010.

[15] A. Song, M. Badiey, V. McDonald, and T. C. Yang, "Time reversal receivers for high rate multiple-input/multiple-output communication," IEEE J. Ocean. Eng., vol. 34, no. 4, pp. 
525-538, Oct. 2011.

[16] D. Green and J. A. Rice, "Channel-tolerant FH-MFSK acoustic signaling for undersea communications and networks," IEEE Journal of Oceanic Engineering, vol. 25, no. 1, pp. 28-39, 2000.

[17] J. Catipovic, M. Deffenbaugh, L. Freitag, and D. Frye, "An acoustic telemetry system for deep ocean mooring data acquisition and control," in OCEANS '89. Proceedings, vol. 3, 1989, pp. 887-892.

[18] K. F. Scussel, J. A. Rice, and S. Merriam, "A new MFSK acoustic modem for operation in adverse underwater channels," in Proceedings of MTS/IEEE OCEANS Conference, vol. 1, 1997, pp. 247-254.

[19] D. Green, "Underwater acoustic communications and networks," in Proc. of the Sixth International Symposium on Underwater Technology, China Ship Scientific Research Center, Apr. 2009.

[20] P. J. Gendron, "Orthogonal frequency division multiplexing with on-off-keying: Noncoherent performance bounds, receiver design and experimental results," U.S. Navy Journal of Underwater Acoustics, vol. 56, no. 2, pp. 267-300, Apr. 2006.

[21] M. Stojanovic, "Low complexity OFDM detector for underwater channels," in Proc. of MTS/IEEE OCEANS Conference, Boston, MA, Sept. 18-21, 2006.

[22] B. Li, S. Zhou, M. Stojanovic, L. Freitag, and P. Willett, "Multicarrier communication over underwater acoustic channels with nonuniform Doppler shifts," IEEE Journal of Oceanic Engineering, vol. 33, no. 2, pp. 198-209, Apr. 2008.

[23] T. Kang and R. Iltis, "Iterative carrier frequency offset and channel estimation for underwater acoustic OFDM systems," IEEE Journal on Selected Areas in Communications, vol. 26, 
no. 9, pp. 1650-1661, Dec. 2008.

[24] G. Leus and P. van Walree, "Multiband OFDM for covert acoustic communications," IEEE Journal on Selected Areas in Communications, vol. 26, no. 9, pp. 1662-1673, Dec. 2008.

[25] S. Zhou and Z.-H. Wang, OFDM for Underwater Acoustic Communications. John Wiley \& Sons, Inc., 2014.

[26] F. Qu and L. Yang, "Orthogonal space-time block-differential modulation over underwater acoustic channels," in Proc. of MTS/IEEE OCEANS Conference, Vancouver, BC, Canada, Sept. 29 - Oct. 42007.

[27] Y. M. Aval and M. Stojanovic, "Differentially coherent multichannel detection of acoustic OFDM signals," IEEE Journal of Oceanic Engineering, 2014; DOI: 10.1109/JOE.2014.2328411.

[28] B. Li, J. Huang, S. Zhou, K. Ball, M. Stojanovic, L. Freitag, and P. Willett, "MIMO-OFDM for high rate underwater acoustic communications," IEEE Journal of Oceanic Engineering, vol. 34, no. 4, pp. 634-644, Oct. 2009.

[29] P. Ceballos and M. Stojanovic, "Adaptive channel estimation and data detection for underwater acoustic MIMO OFDM systems," IEEE Journal of Oceanic Engineering, vol. 35, no. 3, pp. 635-646, Jul. 2010.

[30] J. Huang, S. Zhou, and P. Willett, "Nonbinary LDPC coding for multicarrier underwater acoustic communication," IEEE Journal on Selected Areas in Communications, vol. 26, no. 9, pp. 1684-1696, Dec. 2008.

[31] M. Wetz, W. G. Teich, and J. Lindner, "PAPR reduction methods for noncoherent OFDMMFSK," Proceedings of the 3rd COST Workshop, July 2006.

[32] D. Mestdagh and P. Spruyt, "A method to reduce the probability of clipping in DMT-based 
transceivers," IEEE Transactions on Communications, vol. 44, no. 10, pp. 1234-1238, Oct. 1996.

[33] P. Van Eetvelt, G. Wade, and M. Tomlinson, "Peak to average power reduction for OFDM schemes by selective scrambling," Electronics Letters, vol. 32, no. 21, pp. 1963-1964, Oct. 1996.

[34] R. Bauml, R. Fischer, and J. Huber, "Reducing the peak-to-average power ratio of multicarrier modulation by selected mapping," Electron. Lett., vol. 32, no. 22, pp. 2056-2057, Oct. 1996.

[35] M. Friese, "Multitone Signals with Low Crest Factor," IEEE Transactions on Communications, vol. 45, pp 1338-1344, October 1997.

[36] E. van der Ouderaa, J. Schoukens, J. Renneboog, "Peak Factor Minimization Using a Time-Frequency Domain Swapping Algorithm," IEEE Transactions on Instrumentation and Measurement, vol. 37, pp 145-147, March 1988.

[37] C. R. Berger, S. Zhou, J. Preisig, and P. Willett, "Sparse channel estimation for multicarrier underwater acoustic communication: From subspace methods to compressed sensing," IEEE Transactions on Signal Processing, vol. 58, no. 3, pp. 1708-1721, Mar. 2010.

[38] L. Wan, Z.-H. Wang, S. Zhou, TC Yang, and Z. Shi, "Performance comparison of Doppler scale estimation methods for underwater acoustic OFDM," Journal of Electrical and Computer Engineering, Special Issue on Underwater Communications and Networks, 2012, doi:10.1155/2012/703243.

[39] D. A. Abraham and P. K. Willett, "Active Sonar detection in shallow water using the Page Test," IEEE Journal of Oceanic Engineering, vol. 27, no. 1, pp. 35-46, Jan. 2002.

[40] M. K. Simon and R. Annavajjala, "On the optimality of bit detection of certain digital 
modulations," IEEE Transactions on Communications, vol. 53, no. 2, Feb. 2005.

[41] AquaSeNT OFDM Modem; http://www.aquasent.com/.

[42] L. Wan, H. Zhou, X. Xu, Y. Huang, S. Zhou, Z. Shi, and J.-H. Cui, "Adaptive modulation and coding for underwater acoustic OFDM," IEEE Journal of Oceanic Engineering, vol. 40, no. 2, pp. 327-336, Apr. 2015.

[43] Z.-H. Wang, S. Zhou, J. Catipovic, and P. Willett, "Parameterized cancellation of partialband partial-block-duration interference for underwater acoustic OFDM," IEEE Transactions on Signal Processing, vol. 60, no. 4, pp. 1782-1795, Apr. 2012. 